\title{
REAL CLOSURES OF SEMILOCAL RINGS, AND EXTENSION OF REAL PLACES
}

\author{
BY MANFRED KNEBUSCH
}

Communicated by Alex Rosenberg, June 7, 1972

(A) All rings in this announcement are commutative and with 1. For any ring $K$ we denote by $W(K)$ the Witt ring of nondegenerate symmetric bilinear forms over $K$.

Definition 1. A signature $\sigma$ of $K$ is a ring homomorphism from $W(K)$ to $\boldsymbol{Z}$.

REMARK 1 . If $K$ is a field, the signatures correspond uniquely with the orderings of $K$ [3], [9]. Thus Theorem 1 below generalizes the main results of Artin-Schreier's theory of ordered fields [1].

We consider pairs $(K, \sigma)$ with $K$ a connected ring and $\sigma$ a signature of $K$. There is an obvious notion of a homomorphism $(K, \sigma) \rightarrow(L, \tau)$ between pairs. We say that a homomorphism $\alpha: K \rightarrow L$ of rings is a (connected) covering, if $\alpha$ is the inductive limit of finite etale connected extensions of $K$, as studied in Galois theory. We say that a homomorphism $\alpha:(K, \sigma) \rightarrow$ $(L, \tau)$ is a covering, if $K \rightarrow L$ is a covering.

DEFINITION 2. A real closure of a pair $(K, \sigma)$ is a covering $\alpha:(K, \sigma) \rightarrow$ $(R, \rho)$ such that $(R, \rho)$ does not admit any coverings except isomorphisms.

By Zorn's lemma any pair $(K, \sigma)$ has at least one real closure.

THEOREM 1. Assume $\alpha:(K, \sigma) \rightarrow(R, \rho)$ is a real closure of a pair $(K, \sigma)$ with $K$ semilocal. Let $K_{s}$ denote the universal covering (= separable closure) of $K$.

(1) For any other real closure $\alpha^{\prime}:(K, \sigma) \rightarrow\left(R^{\prime}, \rho^{\prime}\right)$ there exists an isomorphism $\beta:(R, \rho) \Im\left(R^{\prime}, \rho^{\prime}\right)$ with $\alpha^{\prime}=\beta \circ \alpha$.

(2) There does not exist any automorphism of $(R, \rho)$ leaving all elements of $K$ fixed except the identity.

(3) The Galois group of $K_{s} / R$ is a 2-group.

Assume in addition that 2 is a unit in A. Then even the following statements are true:

(3a) $K_{s}=R(\sqrt{-1})$.

(4) If $R^{\prime} / K$ is any covering such that $\left[K_{s}: R^{\prime}\right]=2$, then $K_{s}=R^{\prime}(\sqrt{-1})$ and $W\left(R^{\prime}\right) \cong Z$. In particular $R^{\prime}$ has a unique signature.

Thus if $K$ is semilocal with 2 a unit the signatures of $K$ correspond uniquely with the conjugacy classes of involutions in the Galois group of $K$.

REMARK 2. If $K$ is a Dedekind domain at least statement (1) of Theorem 1 remains true and $\left[K_{s}: R\right] \leqq 2$.

AMS (MOS) subject classifications (1970). Primary 13B05, 12J20; Secondary $15 \mathrm{~A} 63$. 
The proofs of part (2) and (3) of Theorem 1 are essentially contained in [7] (cf. [7a]). Also the proofs of the other parts and of Remark 2 depend on this paper and on [5], [6]. The proof of (3a) and (4) proceeds by imitation of a classical proof of the fundamental theorem of algebra. The main point in the proof of (1) is to prove simultaneously the following trace formula :

THEOREM 2. Let $L$ be a finite covering of the semilocal ring $K$ and let $\operatorname{Tr}^{*}: W(L) \rightarrow W(K)$ denote the transfer map induced by the regular trace $\operatorname{Tr}=\operatorname{Tr}_{L / K}[11]$. Then, for any signature $\sigma$ of $K$ and any $z$ in $W(L)$,

$$
\sigma\left(\operatorname{Tr}^{*}(z)\right)=\sum_{\tau \mid \sigma} \tau(z)
$$

where $\tau$ runs through all signatures of $L$ lying over $\sigma$, with the convention that the sum is zero if there are no such $\tau$.

In [4], a proof of Theorem 1(1) and Theorem 2 over fields has been given which, with the knowledge of the other parts of Theorem 1, immediately generalizes to semilocal rings with 2 a unit. This proof also gives a good idea of the techniques needed for the general case. Of course, no statements about zeros of real polynomials are used (e.g., Sturm's theorem). The connection between Burnside and Witt rings, studied in [2], gives, in the case that 2 is a unit, another approach to Theorem 1(1) [Dress, oral communication].

For any ring $A$, let $2^{-\infty} A$ denote the localization with respect to the multiplicative system of powers of 2 . Theorems 1 and 2 imply

THEOREM 3. Assume that $K \rightarrow L$ is a finite covering of semilocal rings and $K \rightarrow K^{\prime}$ is a homomorphism into a semilocal ring $K^{\prime}$.

(i) $2^{-\infty} W(L)$ is finite etale over $2^{-\infty} W(K)$ and is generated as module over $2^{-\infty} W(K)$ by at most $[L: K]$ elements.

(ii) The kernel and cokernel of the natural map

are 2-torsion groups.

$$
W(L) \otimes_{W(K)} W\left(K^{\prime}\right) \rightarrow W\left(L \otimes_{K} K^{\prime}\right)
$$

(B) For arbitrary valuation rings (at least) it is also possible to study by the same methods the behavior of certain signatures in "ramified coverings". This leads to results about real places of fields. From now on $R$ denotes a fixed real closed field and $K$ denotes a field of characteristic zero.

Definition 3. A signature $\sigma$ of $K$ and a place $\phi: K \rightarrow R \cup \infty$ are compatible, if $\phi(a) \geqq 0$ or $\phi(a)=\infty$ for all $a$ in $K$ which are positive with respect to $\sigma$ (cf. Remark 1 ).

One easily proves that for any $R$-valued place $\phi$ of $K$ there exists at least one signature of $K$ which is compatible with $\phi$. 
THEOREM 4. Assume that $L$ is an algebraic field extension of $K$, that $\tau$ is a signature of $L$ and $\phi$ is an $R$-valued place of $K$, compatible with the restriction $\tau \mid K$ of $\tau$ to $K$. Then there exists a unique $R$-valued place $\psi$ of $L$ extending $\phi$ and compatible with $\tau$.

This theorem refines a result of Lang [8, Theorem 6] which says that any $R$-valued place of $K$ can be extended to an $R$-valued place of at least one real closure of $K$.

For any $a$ in $K^{*}$ we denote as usual by $(a)$ the element of $W(K)$ represented by the one-dimensional symmetric bilinear form with matrix $(a)$. It can be shown by well-known arguments (e.g., [10, Chapter V, proof of Lemma 1.2]) that any place $\phi: K \rightarrow R \cup \infty$ yields a well-defined additive map $\phi_{*}$ from $W(K)$ to $Z$, whose value on an element $(a)$ is obtained in the following way: Let $\rho$ denote the unique signature of $R$. If there is some $b$ in $K$ such that $\phi\left(a b^{2}\right) \neq 0$ and $\neq \infty$ then $\phi_{*}(a)=\rho\left(\phi\left(a b^{2}\right)\right)$ with an arbitrary choice of such an element $b$. Otherwise $\phi_{*}(a)=0$. Clearly a signature $\sigma$ of $K$ is compatible with $\phi$ if and only if $\sigma(a)=\sigma_{*}(a)$ for all $a$ in $K$ with $\phi_{*}(a) \neq 0$.

As a counterpart of Theorem 4 we have

THEOREM 5. Assume that $L$ is an arbitrary field extension of $K$, that $\psi$ is an $R$-valued place of $L$ and $\sigma$ is a signature of $K$ compatible with $\psi \mid K$. There exists a signature $\tau$ of $L$ compatible with $\psi$ and extending $\sigma$ (i.e., $(K, \sigma) \rightarrow(L, \tau)$ is a homomorphism $)$ if and only if $\sigma(a)=\psi_{*}(a)$ for all $a$ in $K_{*}$ with $\psi_{*}(a) \neq 0$.

THEOREM 6. Assume $L_{1}$ is an algebraic field extension of $K$ and $L_{2}$ is an arbitrary field extension of $K$. Further assume that, on each $L_{i}$, an $R$-valued place $\phi_{i}$ is given and $\phi_{1}\left|K=\phi_{2}\right| K$. Then the following are equivalent:

(i) There exists a field composite $F$ of $L_{1}$ and $L_{2}$ over $K$ and an $R$-valued place $\psi$ on $F$ extending both $\phi_{1}$ and $\phi_{2}$.

(ii) $\phi_{1 *}(a)=\phi_{2 *}(a)$ for all $a$ in $K^{*}$ such that both $\phi_{1 *}(a)$ and $\phi_{2 *}(a)$ are not zero.

THEOREM 7. Let $L$ be a finite algebraic field extension of $K$ and $\phi$ an $R$-valued place of $K$. Then, for any $z$ in $W(L)$,

$$
\phi_{*}\left(\operatorname{Tr}_{L / K}^{*}(z)\right)=\sum_{\psi \mid \phi} \psi_{*}(z)
$$

where $\psi$ runs through all $R$-valued places of $L$ extending $\phi$.

Thus $\phi$ has $\phi_{*}\left(\operatorname{Tr}_{L / K}^{*}(1)\right) R$-valued extensions to $L$.

Detailed proofs will appear elsewhere. I wish to thank A. Dress and A. Rosenberg for very helpful discussions. 


\section{REFERENCES}

1. E. Artin and O. Schreier, Algebraische Konstruktion reeller Körper, Abh. Math. Sem. Univ. Hamburg 5 (1926), 85-99.

2. A. Dress, "A connection between Burnside- and Witt-rings," Notes on the theory of representations of finite groups. I, Chap. 2, Appendix B, Univ. Bielefeld, 1971.

3. D. K. Harrison, Wittrings, Lecture Notes, Dept. Math., Univ. Kentucky, Lexington, Ky., 1970.

4. M. Knebusch, On the uniqueness of real closures and the existence of real places, Comment. Math. Helv. (to appear).

5. M. Knebusch, A. Rosenberg and R. Ware, Structure of Witt-rings and quotients of abelian group rings, Amer. J. Math. 94 (1972), 119-155.

6. - Grothendieck- and Witt-rings of hermitian forms over Dedekind rings, Pacific J. Math. (to appear).

7. — Signatures on semi-local rings, J. Algebra (to appear).

7a. - Signatures on semilocal rings, Bull. Amer. Math. Soc. 78 (1972), 62-64.

8. S. Lang, The theory of real places, Ann. of Math. (2) 57 (1953), 378-391. MR 14, 841.

9. J. Leicht and F. Lorenz, Die Primideale des Wittschen Ringes, Invent. Math. 10 (1970), 82-88. MR 42 \# 1851.

10. J. Milnor (with D. Housemoller), Symmetric bilinear forms, Lecture Notes, Inst. Advanced Study, Princeton, N.J., 1971.

11. W. Scharlau, Zur Pfisterschen Theorie der quadratischen Formen, Invent. Math. 6 (1969), 327-328. MR 39 \# 2793.

Mathematische Institut, Universität DES SAarlandes, D-66 SAarbRÜCKen IM Stadtwald, Federal Republic OF Germany 